•综述・

\title{
榕-传粉榕小蜂非一对一共生关系的研究进展
}

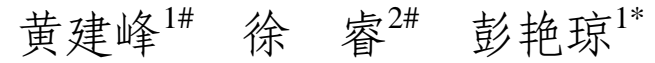 \\ 1 (中国科学院热带森林生态学重点实验室, 中国科学院西双版纳热带植物园, 云南预腊 666303) \\ 2 (云南省热带作物科学研究所, 云南景洪 666100)
}

摘要：榕-传粉榕小蜂系统是研究动植物专性互惠共生关系的经典体系。早期形态学研究认为它们之间遵循一对 一原则, 即 1 种榕树只有 1 种榕小蜂为其传粉, 而 1 种传粉榕小蜂也只能在 1 种榕树上繁殖后代。然而随着研究的深 入, 报道了越来越多非一对一共生关系的案例, 尤其是隐存种的发现, 打破了一对一原则在榕-传粉榕小蜂系统中 的普适性。非一对一共生关系包括多种传粉者共现于 1 种宿主榕树和多种榕树共享 1 种传粉者两种情况，有各自不 同的发生机制和规律。本文从形态学描述阶段、多学科综合证据阶段和传粉榕小蜂隐存种的发现 3 个阶段综述了 榕-传粉榕小蜂非一对一共生关系的研究进展, 并对其所引发的科学问题进行了探讨。多种传粉者共现于 1 种宿主 榕树的情况, 使得榕小蜂的种间杂交成为可能; 而多种榕树共享1种传粉者, 为宿主榕树的种间杂交提供了机会。 然而, 杂交事件的检出率却很低, 暗示存在明显的生殖隔离。另外, 传粉者共现和共享传粉者两种情况在不同性系 统榕树上的发生率和发生机制存在明显差异, 暗示不同性系统榕树的宿主专一性不同。大量小蜂隐存种的发现, 引发了对其成种机制、共现机制的研究, 以及对小蜂生物多样性的重新评估, 并给人工控制实验的开展带来了新 的问题。每个榕-传粉榕小蜂组合都具有其独特的协同进化历史, 为研究动植物间的协同进化机制和规律提供了丰 富素材。对榕-传粉榕小蜂专性互惠共生关系的形成、维持和打破机制开展综合研究, 才能充分认识该系统，也有 助于认识动植物间的协同进化过程和规律。

关键词：榕树；传粉榕小蜂；一对一原则；隐存种；传粉者共现；共享传粉者

\section{Progress on the breakdown of one-to-one rule in symbiosis of figs and their pollinating wasps}

Jianfeng Huang ${ }^{1 \#}$, Rui $\mathrm{Xu}^{2 \#}$, Yanqiong Peng ${ }^{{ }^{*}}$

1 Key Laboratory of Tropical Forest Ecology, Xishuangbanna Tropical Botanical Garden, Chinese Academy of Sciences, Mengla, Yunnan 666303

2 Yunnan Institute of Tropical Crops, Jinghong, Yunnan 666100

\begin{abstract}
The fig-fig-pollinating wasp symbiosis provides a model system for investigating the mutualistic interaction between plants and animals. A simple one-to-one rule was proposed to describe the highly specialized reciprocal relationships between figs and their species-specific pollinating fig wasps based on the initial studies: each fig tree species is obligatorily pollinated by one fig wasp species, and each wasp species can only reproduce in one fig species. With the deepening of research, however, increasing cases of breakdown in species-specificity have been reported, especially the reveal of cryptic pollinating fig wasp species progressively weaken the universality of one-to-one rule in fig-fig-pollinating wasp symbiosis. The documented cases of breakdown in the one-to-one rule were divided into two types, including copollinator and pollinator sharing, which have their own different mechanisms. Pollinator sharing is pollination of different species of Ficus by the same pollinator, while copollinator is the co-occurrence of more than one species of pollinators in one host fig. Here, the research progress on the breakdown of one-to-one rule is presented in three stages, i.e. morphological description, multidisciplinary evidence and discovery of cryptic pollinating wasp species. Some future challenges and research prospects resulted from the breakdown of one-to-one rule
\end{abstract}


are outlined. Copollinators may lead to the interspecific hybridization between pollinator species, while pollinator sharing may result in the hybridization of host figs. Nonetheless, the hybridization in fig-fig-pollinating wasp symbiosis is very rare and suggests a strong level of reproductive isolation. Furthermore, the incidence and pattern of pollinator sharing and copollinator are differentiated in dioecious and monoecious figs, which suggests a differentiated host-specificity. The identification of cryptic species triggers the research of its speciation and co-occurrence, as well as the reassessment of pollinator biodiversity. Failing to recognize cryptic species also will limits the effectiveness of the controlled experiment in fig-fig-pollinating wasp symbosis. Each pair of fig and pollinating wasp possesses its distinctive coevolutionary history, and there are more than 750 pairs across the globe, which provide rich materials to explore the coevolutionary process and mechanism between plants and animals. The integrated study of formation, maintenance and breakdown mechanisms of the host-specificity will contribute to the understanding of fig-fig-pollinating wasp mutualism, and the coevolutionary process and mechanism between plants and animals.

Key words: fig; fig pollinating wasp; one-to-one rule; cryptic species; copollinator; pollinator sharing

协同进化塑造着种间关系，影响着生态系统的 结构和功能及其动态。榕树及其传粉榕小蜂构成的 互惠共生系统(以下简称榕-传粉榕小蜂系统)早在 7,500 万年前就已建立, 是动植物间最紧密的协同 进化关系之一(Cruaud et al, 2012)。榕树是榕属 (Ficus) 植物的总称, 隶属于桑科, 全球约 750 种 (Berg, 1989)。榕树有乔木、灌木、攀援藤本等丰富 的生活型, 占据雨林中的乔木层、灌木层、藤本层 (Harrison, 2005), 一些榕树还具有气根、板根、绞杀、 独木成林、老茎生花等独特现象。榕树为多种动物 提供了食物和栖息地, 被公认为热带雨林的关键类 群(许再富, 1994; Shanahan et al, 2001)。

所有的榕树均具隐头花序, 隐头花序也被称为 榕果, 其顶部有一个由苞片组成的苞片口, 是榕果 与外界交流的唯一通道(Janzen, 1979)。这种特殊结 构使得榕树需要依赖专性的传粉榕小蜂才能完成 有性生殖; 同时隐头花序也为传粉榕小蜂提供产 卵、繁衍后代的场所。从高达数十米的大乔木到低 矮的匍匐藤本, 榕树都依赖大小只有 $1-3 \mathrm{~mm}$ 、成虫 期只有1-2天的专性传粉榕小蜂传粉, 两者在形态、 生理、生态、行为等方面都达到了高度适应。这种 相互依赖、不可或缺的关系被Wiebes (1963)用“一对 一”原则(one-to-one rule)来描述。该原则被认为在榕 -传粉榕小蜂系统中具有普适性, 即 1 种榕树只有 1 种传粉榕小蜂为其传粉, 而1种传粉榕小蜂也只能 在1种榕树上繁殖后代。这种高度专性互惠共生关 系的形成和维持成为一个自然之谜, 吸引了众多国 内外学者去探索。然而, 对这种一对一关系的质疑 一直存在。随着技术和研究方法的革新, 发现了越 来越多打破一对一原则的案例, 包括多种传粉者共
现于 1 种宿主榕树和多种榕树共享 1 种传粉者两种 情况。正如其他新观点、新理论的出现一样, 榕传粉榕小蜂系统非一对一共生关系的观点也经历 了早期的反对和质疑、实验的反复验证，再到最后 被接受三个阶段。

\section{形态学描述阶段}

早在1886年, Müller就记录了多种榕树共享传 粉榕小蜂 Blastophaga brasiliensis 的情况 (Müller, 1886)。Grandi (1916)报道了小蜂Eupristina saundersi 为印度境内菩提树 (F. religiosa)传粉的情况, 但同时 也发现菩提树还存在另一种传粉小蜂, 即分布于马 来西亚(Mayr, 1885)、印度、斯里兰卡(Grandi, 1922; Joseph, 1953; Johri \& Konar, 1955)和以色列(Wiebes, 1963)的Platyscapa quadraticeps。后来的学者认为 Grandi (1916)的报道在宿主鉴定方面存在错误, 因 为 E. saundersi的雄虫发现于 F. retusa var. nitida (Wiebes, 1963; Rasplus, 1996)而非菩提树。Grandi (1927)还报道了菲律宾群岛分布的F. ulmifolia和F. heterophylla共享传粉者Blastophaga browni的情况, 但进一步研究发现菲律宾群岛并没有 $F$. heterophylla分布, 最初被认为是F. heterophylla的标 本均属于F. ulmifolia (Wiebes, 1963)。基于形态特征 描述榕一传粉榕小蜂非一对一共生关系的报道还很 多, Wiebes (1963)总结了打破一对一原则的案例, 认为这些案例基本上都源于宿主或传粉榕小蜂种 类鉴定错误, 并不可信。

一年之后, Wiebes (1964) 报道了 Ceratosolen arabicus和C. galili两种小蜂共现于非洲榕树植物F. sycomorus的情况。其中C. arabicus是有效传粉者, 
为F. sycomorus主动传粉; 而C. galili有发达的花粉 筐, 但无花粉刷, 缺乏主动传粉行为, 是一种只寄 生不传粉的欺骗者 (Galil \& Eisikowitch，1968, 1969)。C. galili能独自占据F. sycomorus一半以上的 榕果, C. arabicus独占榕果的比率相对较低, 而两者 共现的榕果约占 $13 \%$ 。但C. galili繁殖的后代数量比 C. arabicus少, 而且个体较小, 这可能是C. galili进 化为欺骗者所付出的代价(Compton et al, 1991)。基 于 $C y t b$ 基因开展的研究显示宿主转移(host shift)是 $C$. galili进化为欺骗者的机制(Kerdelhué et al, 1999)。进 一步研究发现, 虽然传粉者C. arabicus和欺骗者C. galili分布区重叠, 但两者在生理和行为上有显著差 异: C. arabicus成虫期寿命较长, 耐高温、干旱和饥 饿的能力强, 并在白天飞行寻找接受期榕果; 而 $C$. galili抗逆能力差, 在夜晚飞行活动, 恶劣的气候和 环境条件对这类欺骗者的影响比对传粉者更大 (Warren et al, 2010)。这种欺骗者类似于非传粉的寄 生榕小蜂, 与传粉榕小蜂在功能、生理、生态等多 方面存在分化。类似的情况也存在于高榕 $(F$. altissima) (Peng et al, 2008, 2010) 和小叶榕 (F. microcarpa) (Martinson et al, 2014)。Boucek等(1981) 认 为 Ellsabethiella stuckenbergi 、Alfonsiella brongersmai和 $A$. longiscapa 共现于 $F$. thonningii的情 况可能也是类似的机制。

Ramírez (1970a)报道了小蜂Pegoscapus mariae 存在于F. tuerckheimii和F. jimenezi两种榕树的情况。 但F. jimenezi的成熟榕果中只发现了其专性传粉者P. jimenezi的后代, 未发现P. mariae小蜂后代, 说明P. mariae虽然能够进入F. jimenezi榕果, 但不能成功繁 殖。另外, Ramírez (1970b)在委内瑞拉境内一株榕树 F. turbinata上发现，5\%的榕果被其他非专性传粉榕 小蜂拜访, 并能成功繁殖小蜂后代、产生种子, 但 种子不能萌发, 而其专性传粉者拜访的榕果所产生 的种子具有 $100 \%$ 的萌发率。上述两例打破一对一原 则的现象, 只是由于传粉者偶然的误访, 并不能成 功繁殖小蜂后代或产生可育的宿主种子, 并未真正 打破一对一原则。

由于早期研究基于形态和野外观察, 有时还存 在鉴定错误(Grandi，1916，1927); 或因凭证标本丢 失而不能核实(Wiebes, 1963); 或因自然界偶然发生 传粉榕小蜂误访, 但不能产生小蜂后代和可育种子 (Wiebes, 1970a, b); 或因共现的传粉榕小蜂中只有1
种为宿主传粉, 而其他种类为不传粉的寄生者或欺 骗者(Galil \& Eisikowitch, 1968, 1969)等, 所以打破 一对一原则的观点并未得到普遍认可。

\section{多学科综合证据阶段}

首例被学者们广泛接受的传粉者打破一对一 原则的实例报道于1985年。分布于非洲中西部加蓬 地区的榕树 F. ottoniifolia 有 2 种传粉榕小蜂, 即 Courtella camerunensis和C. gabonensis。它们都具花 粉筐, 能为宿主主动传粉, 并产生可育种子和小蜂 后代; 两者同域分布，甚至能生存于同一榕果 (Michaloud et al, 1985)。但它们的生境偏好不同: C. camerunensis偏爱郁闭的雨林生境，此生境中 $57 \%$ 的F. ottoniifolia榕果被其独占，只有 $20 \%$ 的榕果被C. gabonensis独占; 而C. gabonensis偏爱开阔生境(例 如农田或村落边), 其中 $68 \%$ 的榕果被其独占，只有 $6 \%$ 的榕果被C. camerunensis独占; 在过渡的森林边 缘地带, 榕果中2种小蜂的数量呈现此消彼长的格 局; 但从雨林向开阔生境过渡的过程中, 榕果中 $C$. camerunensis小蜂数量显著减少, 而C. gabonensis数 量缓慢增加(Michaloud et al, 1985, 1996)。但无论在 哪种生境中, 同一株榕树上几乎都存在 2 种小蜂, 并且 23-26\%的榕果有 2 种小蜂 (Michaloud et al, 1996)。 Michaloud 等 $(1985,1996)$ 还报道了 Ceratosolen capensis、C. flabellatus和C. silvestrianus 共现于非洲榕树 F. sur的情况。3种小蜂均能够为 F. sur主动传粉, 并产生种子和小蜂后代(Kerdelhué et al, 1997)。其中, C. capensis为非洲大陆南部和东北 部分布的F. sur传粉, C. flabellatus和C. silvestrianus 为非洲西至中东部的 $F$. sur传粉。在非洲西部, $C$. flabellatus和C. silvestrianus可存在于同株榕树, 但 几乎不共现于同一榕果。从更大范围来看, 非洲西 部喀麦隆境内海拔较高的Buea、Bamenda高地以及 Monts Mandara地区的F. sur 只有C. silvestrianus为其 传粉(Michaloud et al, 1996)。生境偏好和异域分布 能够有效避免种间竞争, 这可能是上述两个案例发 生传粉者共现的重要机制。基于此，Michaloud等 (1996)提出了异域成种(allopatric speciation)和宿主 转移 2 个途径或假说来解释传粉者共现现象。

随着3部关于榕-传粉榕小蜂系统专著的发表 (Wiebes \& Compton, 1990; Berg \& Wiebes, 1992; Wiebes, 1994), 越来越多打破一对一原则的案例被 
报道。例如, Berg和Wiebes (1992)发现在非洲的105 种榕树中，24种存在非一对一共生关系; Wiebes (1994)在亚洲和澳洲分布的175种榕树中，发现11种 打破一对一原则的案例。Rasplus (1996)进一步指出, 在已知的榕-传粉榕小蜂组合中, 约有 $13 \%$ 的组合 不符合一对一原则，其中非洲 $17 \%$ 的榕树种类具有 至少 2 种传粉榕小蜂，约 $15 \%$ 的小蜂物种存在于 2 种 或2种以上的宿主榕树; 这个比例在澳大拉西亚地 区分别为 $13 \%$ 和 $7 \%$ 。Cook和Segar (2010)总结了 142 种雌雄同株榕树的研究, 发现其中45种存在多种传 粉者, 比例高达 $32 \%$ 。

相对而言, 共享传粉者的案例比较少见(Rasplus，1996)。木瓜榕(F. auriculata) 和苹果榕 (F. oligodon)共享传粉榕小蜂Ceratosolen emarginatus 的情况很早就被提及(Wiebes, 1963), 它们和海南榕 (F. hainanensis) 以及北碚榕(F. beipeiensis)均属于木 瓜榕复合体(F. auriculata complex), 不少学者对它 们的分类地位存在疑问(Corner, 1965; Berg \& Corner, 2005), 最新的分子证据建议把它们合并为一个 种(Zhang et al, 未发表数据)。Rasplus (1996)报道了 小蜂Ellsabethiella socotrensis为索科特拉岛、埃塞俄 比亚和沙特阿拉伯境内的F. vasta, 以及肯尼亚和赞 比亚境内的F. wakefieldii传粉的情况; 后来发现该 种小蜂还为非洲南部的F. natalensis传粉(Cornille et al, 2011)。Cornille等(2011)还发现F. natalensis与 $F$. burkei共享小蜂E. stuckenbergi, 并发现这 2 种榕树 的接受期榕果能够释放高度相似的化学挥发物, 这 被认为是吸引小蜂发生宿主转移从而为 2 种宿主传 粉的重要机制。异形花榕(F. heterostyla)和肉托榕(F. squamosa)为 2 种分布于亚洲热带大陆的雌雄异株榕 树, 刘桂香(2013) ${ }^{\circledR}$ 和Liu等(2015)发现分布于我国 西双版纳地区的异形花榕和肉托榕共享1种未描述 的传粉榕小蜂新种Ceratosolen sp.。该种小蜂能够为 2 种榕树主动传粉, 并成功繁殖小蜂后代。2种榕树 在西双版纳地区虽同域分布, 但偏爱生境不同。肉 托榕为小灌木, 偏爱生长于水流两岸或裸露于水面 的岩石缝中, 雨季洪水期常被浸泡于流水中, 水流 传播被认为是其种子的主要传播方式(Pothasin et al, 2016); 异形花榕为小乔木, 偏爱生长于次生林边缘 和道路两旁。物候调查发现, 肉托榕结果期集中在

(1) 刘桂香 (2013) 榕树与传粉榕小蜂非一对一关系演化的生态学机制. 硕士学位论文, 中国科学院大学, 北京.
干季, 雌雄株均在3月达到结果高峰; 分布于河流 边的肉托榕在雨季的7-9月会淹没于洪水中, 不结 果。异形花榕种群全年都有树结果, 7月份达到结果 高峰, 2-3月结果量最少。这种互补的结果物候被认 为是传粉榕小蜂在同域分布的 2 种榕树之间发生宿 主转移的重要原因(刘桂香, 2013 ${ }^{\circledR}$; Liu et al, 2015)。

与早期随机性很强的基于野外观察和形态分 类不同, Michaloud等(1985)的研究开启了综合植物 学、昆虫学、分类学、生物化学、生态学、物候学 等多学科证据来研究非一对一共生关系的新局面。 越来越多的案例和证据使得学者们开始逐渐接受 打破一对一原则的真实存在, 但质疑的声音仍在。 例如, Wiebes (1994)仍然质疑传粉者共现现象, 指 出更深入的分类学研究也许会证明具有 2 种或多种 传粉者的榕树其实应该被进一步划分为 2 个或多个 种或者亚种、变种等, 在榕树种或者种以下水平有 各自专一的传粉者。

\section{传粉榕小蜂隐存种的发现}

分子遗传标记技术的发展给榕树和传粉榕小 蜂共生关系研究带来了革命性改变。理论上讲, 如 果榕树和传粉榕小蜂之间存在严格的协同成种 (cospeciation)和种水平上的一对一协同进化关系, 那么两者在系统发育树上应表现为一一对应的关 系。然而事实并非如此。虽然早期的分子系统学研 究(Herre et al, 1996; Machado et al, 2001; Weiblen, 2001; Weiblen \& Bush, 2002)显示, 榕树与传粉榕小 蜂具有较一致的系统发生关系, 但这些研究往往以 少数甚至 1 个物种代表 1 个组或亚组, 忽略了高级分 类阶元可能不是单系的问题(Rønsted et al, 2008); 以及仅用 1 个个体代表 1 个物种, 忽略隐存种的存在 (Su et al, 2008)。㱠如，Su等(2008)通过对每个物种进 行多样本采集而开展的系统发育研究发现了非一 对一共生关系的存在。Cruaud等(2012)对全球范围 内收集的近 200 个榕-传粉榕小蜂物种开展的分子 系统学研究, 发现了大量宿主转移和重复成种事 件。然而, 对一对一原则更为严竣的挑战来自于种 群遗传学的研究和大量传粉榕小蜂隐存种的发现。

关于传粉榕小蜂隐存种的报道最早可追溯到 Molbo等(2002)的研究。他们从巴拿马多株榕树植物 F. obtusifolia上收集了 800 个小蜂样本, 它们在形态 上都属于Pegoscapus hoffmeyeri, 而微卫星(simple 
sequence repeats, SSRs) 数据支持存在 2 个 $P$. hoffmeyeri隐存种。之后, Molbo等(2003)又对分布于 巴拿马运河附近的 8 种榕树植物的传粉小蜂进行了 采样, 发现来自于同种榕树的不同小蜂个体均属于 同一形态种(morphospecies), 且均属于Pegoscapus 属, 支持一对一原则; 而核基因微卫星数据分析显 示, 其中 4 个形态种均存在 2 个隐存种; 线粒体基因 COI分析显示, 同一形态种下不同隐存种之间的遗 传分化大(4.2-6.6\%)。结合COI的变异速率推算, 这 些隐存种早在1.5-5.1百万年之前就已形成。同时, 还检测到榕树植物F. obtusifolia的 2 个小蜂隐存种之 间存在低频率的杂交, 但未发现回交和基因渗入信 号。高达 $50 \%$ 的发生率显示隐存种的出现并非偶然, 至少在Pegoscapus属中具有普遍性。值得一提的是, 该研究在作为外类群的小蜂Tetrapus americanus中 也检测到了隐存种, 而Tetrapus属被认为是传粉榕 小蜂中最原始的类群(Machado et al, 2001; Weiblen, 2001; Weiblen \& Bush, 2002; Cruaud et al, 2010), 暗 示隐存种反复出现于榕-传粉榕小蜂系统的协同进 化历史中。同时, COI数据还显示, 有些形态种(例如 F. obtusifolia的传粉者P. hoffmeyeri)的 2 个隐存种为 姊妹种关系, 暗示它们起源于同一宿主; 而另外一 些形态种(例如F. popenoei的传粉者P. gemellus)的2 个隐存种明显不为姊妹种, 表明其中1个隐存种必 然来自于宿主转移。P. gemellus的 1 个隐存种被F. popenoei和F. bullenei共享的现象，也进一步支持了 宿主转移的存在, 但可能还处于宿主转移早期阶段 (Molbo et al, 2003), 导致它在2个宿主间未出现明 显的形态分化。

通过对澳洲东部沿海18个自然分布点和美国 圣地亚哥1个引种点的F. rubiginosa传粉榕小蜂开展 研究, Haine等(2006)发现形态上属于Pleistodontes imperialis的小蜂样本, 在 $C y t b$ 基因树上形成 4 个独 立分支(Clades 1-4)。分支间遗传分化(9-17\%)远大 于分支内分化(0-7\%), 而形态上能够明显区分的 Pleistodontes属下种间的遗传分化为10-26\%, 支持 4 个不同分支为 4 个隐存种, 并表现为姊妹种关系, 它们在4.4-7.1百万年前发生物种分化。Clade 1广布 于整个分布区，也是唯一出现在引种区的类型; Clade 2限于昆士兰北部Townsville地区, Clade 3限 于昆士兰北部, Clade 4广布于昆士兰。昆士兰境内 的多数采样点均发现有 2 个或以上的隐存种。所有
样本均检测到Wolbachia感染, 为3个Wolbachia菌系 (W1-3)。Clade 3被菌系W1感染, Clade 1, 2, 4同时被 W2和W3感染。Wolbachia感染和地理隔离一定程度 上促进了传粉榕小蜂的物种分化, 但难以解释昆士 兰地区不同隐存种的分化和共现机制。在Haine等 (2006)基础上, Darwell等(2014)进行了更广泛的采 样, 发现了第5个P. imperialis隐存种。Sutton等(2017) 进一步综合 COI和 Cytb 基因以及核基因微卫星数据, 对Townsville地区的P. imperialis种群开展了研究。 结果支持该地区存在3个隐存种, 其中 $13 \%$ 的榕果 有 2 个或全部 3 个隐存种。虽然检测到几个疑似杂交 个体, 但未发现基因渗入证据, 显示共现的隐存种 之间存在明显的生殖隔离。Moe和Weiblen (2010)在 亚洲热带至澳洲北部采集了6种榕树的传粉榕小蜂 样本，发现同种榕树不同分布区采集的小蜂样本均 属于同一形态种。然而, COI数据显示 6 个形态种内 均存在隐存种, 不同的隐存种有各自独立的分布区, 表明异域成种可能是其隐存种分化的重要机制。

国内学者对榕小蜂隐存种也开展了相关研究。 Lin等(2011)对台湾岛分布的棱果榕(F. septica)传粉 榕小蜂Ceratosolen bisulcatus进行了样本采集, 根 据体表颜色将其分为黑色和黄色两类，黑色类型广 布于台湾岛, 而黄色类型只发现于台湾岛南部。南 部采样点中, 两类小蜂共现于 $27 \%$ 的榕果, 黑色和 黄色各独占 $38 \%$ 和 $35 \%$ 的榕果。而COI数据显示小蜂 分化为 3 个强烈支持的单系分支, 黄色类型为 1 支, 黑色类型分化为2支，2支之间遗传分化较大(7.2\%), 明显属于 2 个隐存种。核基因微卫星数据也支持 3 个 分支之间存在显著分化(Lin et al, 2011)。Sun等(2011) 从海南和福建不同分布点的 9 株小叶榕上采集了 67 个小蜂样本, 它们在形态上均属于 Eupristina verticillata, 而COI和ITS2序列支持存在3个隐存种。 三者分布区没有明显隔离, 但其中 2 个隐存种分别 被不同的Wolbachia菌系感染, 第3个隐存种未被感 染。分子钟推算显示, 2种Wolbachia菌系的分化时间 要早于对应的 2 种小蜂隐存种的分化时间, 暗示 Wolbachia感染可能诱导了小蜂的分化(Sun et al, 2011)。Chen等(2012)对分布于中国东南部的薜荔(F. pumila var. pumila)传粉者Wiebesia pumilae进行了 研究, 发现形态上归于 W. pumilae的样本显著地分 化为 3 个分支，分支间 COI 基因的遗传分化(7.2$11.6 \%)$ 远大于分支内分化(0.6-0.8\%), 符合根据条 
形码COI划分为不同物种的标准(Hebert et al, 2003, 2004), 但宿主榕树植物却没有相应的分化。不同隐 存种在邻接分布区存在同株共现现象, 但几乎不能 共现于同一榕果, 从大的范围上来看, 表现为隔离 分布格局(Chen et al, 2012; 刘敏, 2014), 生殖隔离 明显, 未出现隐存种杂交情况(Liu et al, 2014)。进 步研究发现同域分布的不同W. pumilae隐存种之间 存在物候分化, 特别是后代小蜂在羽化和飞出榕果 的时间上存在分化, 这可能是其共现和生殖隔离的 重要机制(Liu et al, 2014)。Yang等(2015)对西双版纳 热带雨林地区1.5 $\mathrm{km}^{2}$ 范围内15种榕树的传粉榕小 蜂进行了样本采集, COI和28S序列分析显示垂叶榕 (F. benjamina)、青藤公(F. langkokensis)和鸡嗉子榕 (F. semicordata) 各自的小蜂形态种均包含 2 个隐存 种。Yang等(2015)进一步采用整合分析 (meta-analysis), 综合已发表数据, 对119对榕-传粉榕小蜂组 合(89组雌雄同株种类, 30组雌雄异株种类)进行分 析, 结果显示其中 41 种榕树存在多种传粉者, 其发 生率在雌雄同株(31.5\%)和雌雄异株榕树(43.3\%)之 间无显著差异。然而共现于雌雄异株榕树的不同传 粉者之间均表现为姊妹种关系, 这一比例在雌雄同 株榕树中仅为 $32.1 \%$, 暗示榕树的性系统不同, 其 传粉者共现现象的发生机制也不同。据此Yang等 (2015)提出：雌雄异株榕树的不同传粉者主要来自 于重复成种，而雌雄同株榕树的不同传粉者多来源 于宿主转移。

\section{4 研究展望}

共现的不同传粉者之间在不同的榕-传粉榕小 蜂组合中进化出了不同的受精前隔离机制, 包括地 理隔离(Michaloud et al, 1996; Haine et al, 2006; Moe \& Weiblen, 2010; Chen et al, 2012)、行为隔离(Galil \& Eisikowitch, 1968, 1969; Warren et al, 2010)、生态 隔离(Michaloud et al, 1996; Chen et al, 2012)、生理 隔离(Haine, 2006; Sun et al, 2011)等。虽然共现的隐 存种小蜂之间有疑似杂交个体被检出, 但未发现基 因渗入信号(Molbo et al, 2003; Sutton et al, 2017), 暗示还存在明显的受精后隔离, 但受精后的隔离机 制还需要更深入的研究。

共享传粉者现象可能导致不同宿主间的杂交 和基因渐渗, 但在不同榕-传粉榕小蜂组合中表现 不同。例如, 在一些案例中未检测到榕树杂交信号
(Ramírez, 1970b), 而在另外一些案例中检测到了杂 交信号和杂交种(Parrish et al, 2003; Tsai et al, 2015), 显示不同榕树类群之间存在不同程度的生殖隔离。 目前对榕树种间杂交的研究很少，对其种间存在的 生殖隔离机制也不清楚。共享传粉者的不同宿主榕 树如何维持自身遗传的独立性，以及宿主的种间杂 交和基因渐渗，必将成为未来重要的研究方向。

小蜂隐存种的大量出现, 开启了对包括隐存种 的成种机制、共现机制的研究以及生物多样性的重 新评估，同时对榕小蜂控制实验的开展也带来了新 的问题。同一株甚至同一榕果内发现不同隐存种, 说明传粉榕小蜂与宿主之间并非同时发生分化并 协同成种，其具体成种机制应该更为复杂。共现于 同一榕果的不同隐存种在形态功能上高度相似，关 系近缘，占据相似的生态位，对其共现机制的研究 也必然成为未来研究的重要方向之一。有研究 (Zhang \& Hanski, 1998; Zhang et al, 2004)提出“性比 率选择” 共存机制, 来解释这种隐存种的疑似共存 现象，对物种共存进行了理论探讨。该理论综合考 虑种群的数量动态和进化动态, 只要求稀有种的性 比率比常见种更偏雌, 而不需要任何形式的生态位 分化, 但还未得到验证, 最大的困难在于难以确定 共现的不同隐存种是否占据同一生态位。同时，小 蜂隐存种的高频率出现也提醒我们: (1)仅仅 1 个或 少数几个小蜂样本不能代表整个榕-传粉榕小蜂系 统; (2)榕小蜂和其他昆虫的物种多样性可能被严重 低估(Cook \& Rasplus, 2003; Bruno \& Cardinale, 2008; Cook \& Segar, 2010)。此外, 隐存种的存在表 明，仅采用形态特征进行物种鉴定具有很大的局限 性, 这对开展人工控制实验带来了诸多困难。榕一 传粉榕小蜂组合适合开展人工控制实验，用于检验 生态学、进化生物学等学科的重要理论, 而隐存种 的存在可能会降低这些控制实验的有效性(Bickford et al, 2007), 须引起注意。

榕树有雌雄同株和雌雄异株之分，早期研究发 现传粉者共现多发生于雌雄同株榕树(Cook \& Segar, 2010), 然而这些研究集中在只有雌雄同株榕 树分布的美洲和非洲地区，存在抽样不平衡的问 题。后来对亚洲分布的雌雄异株榕树开展研究，同 样发现了传粉者共现现象(Lin et al, 2011; Chen et al, 2012; Yang et al, 2015), 其发生率在雌雄同株和雌 雄异株榕树之间并无显著差异，但发生机制存在差 
异(Yang et al, 2015)。而共享传粉者的报道较少，主 要发生于雌雄同种榕树, 在雌雄异株榕树上的发生 率较低(Cook \& Segar, 2010; Moe et al, 2011)。在已 报道的25例共享传粉者案例中, 有17例发生于雌雄 同株榕树, 是雌雄异株榕树的 2 倍 (Wachi et al, 2016)。雌雄异株榕树的不同传粉者多为姊妹种关系, 且具有更低的共享传粉者发生率, 暗示雌雄异株榕 树传粉者的宿主专一性要高于雌雄同株榕树传粉 者。Yang等(2015)提出了2个假设来解释这种现象: (1)雌雄异株榕树具有更强的受精前隔离机制, 主要 表现在传粉者对雌雄异株榕树物种的榕果挥发物 具有更高区分度; (2)雌雄异株榕树传粉者发生宿主 识别错误需要付出更高的适合度代价(fitness cost)。 因此, 宿主转移事件不太容易发生于雌雄异株榕树, 但还需要更多的研究进行验证。未来对榕果挥发 物、传粉榕小蜂的化学感受器(主要是触角)以及气 味结合蛋白、化学感受蛋白等在榕-传粉榕小蜂系 统中的演化机制和规律开展研究, 对于认识其共生 关系的专性程度和进化具有重要意义。

\section{结语}

本文分 3 个阶段综述了榕-传粉榕小蜂系统非 一对一共生关系的研究进展, 并对未来研究进行了 展望。虽然一对一原则在某些榕-传粉榕小蜂组合 中依然适用，但研究的深入、技术的革新以及数据 的积累, 逐步打破了一对一原则的普适性。一对一 和非一对一共生关系同时存在, 表明严格和发散两 种协同进化模式并存于榕-传粉榕小蜂系统(陈艳等, 2010)。通过对榕树及其传粉者一对一共生关系的建 立、维持以及打破机制的综合研究才能让我们越来 越接近这对生死相依的物种间关系的真相。榕树与 其传粉者之间的协同进化并不是严格、同步的简单 重复过程。每一个榕-传粉榕小蜂组合都有其独特 性，都有各自特有的协同进化机制。750余种榕树意 味着750余种协同进化关系的变体, 为研究进化生 物学的一些基本问题和理论提供了优良的实验模 型和丰富的素材。全球丰富多彩的生物类型是生物 协同进化的结果, 通过对榕-传粉榕小蜂系统开展 研究, 对探讨动植物之间的种种协同作用, 正确认 识生物界内在的关系具有重要意义; 对包括具有专 一性协同作用的丝兰-丝兰蛾、算盘子-头细蛾等体 系, 以及自然界普遍存在发散性协同作用的生物类
群之间的种种相互关系都具有重要的参考价值。

\section{参考文献}

Berg CC (1989) Classification and distribution of Ficus. Experientia, 45, 605-611.

Berg CC, Corner EJH (2005) Moraceae (Ficus). In: Flora Malesiana (ed Nooteboom HP), pp. 340-342. National Herbarium of the Netherlands, Leiden.

Berg CC, Wiebes JT (1992) African fig trees and fig wasps. Verhandelingen der Koninklijke Nederlandsche (Nederlandse) Akademie van Wetenschappen, 89, 1-298.

Bickford D, Lohman DJ, Sodhi NS, Ng PK, Meier R, Winker K, Ingram KK, Das I (2007) Cryptic species as a window on diversity and conservation. Trends in Ecology \& Evolution, 22, 148-155.

Boucek Z, Watsham A, Wiebes JT (1981) The fig wasp fauna of the receptacles of Ficus thonningii (Hymenoptera, Chalcidoidea). Tijdschrift Voor Entomologie, 124, 149-233.

Bruno JF, Cardinale BJ (2008) Cascading effects of predator richness. Frontiers in Ecology and the Environment, 6, 539-546.

Chen Y, Compton SG, Liu M, Chen XY (2012) Fig trees at the northern limit of their range: The distributions of cryptic pollinators indicate multiple glacial refugia. Molecular Ecology, 21, 1687-1701.

Chen Y, Li HQ, Liu M, Chen XY (2010) Species-specificity and coevolution of figs and their pollinating wasps. Biodiversity Science, 18, 1-10. (in Chinese with English abstract) [陈艳，李宏庆，刘敏，陈小勇 (2010) 榕-传粉榕小蜂间 的专一性与协同进化. 生物多样性, 18, 1-10.]

Compton SG, Holton KC, Rashbrook VK, van Noort S, Vincent SL, Ware AB (1991) Studies on Ceratosolen galili, a non-pollinating Agaonid fig wasp. Biotropica, 23, 188-194.

Cook JM, Rasplus JY (2003) Mutualists with attitude: Coevolving fig wasps and figs. Trends in Ecology \& Evolution, 18, 241-248.

Cook JM, Segar ST (2010) Speciation in fig wasps. Ecological Entomology, 35, 54-66.

Corner EJH (1965) Check-list of Ficus in Asia and Australasia, with keys to identification. The Gardens' Bulletin Singapore, 21, 1-186.

Cornille A, Underhill JG, Cruaud A, Hossaert-McKey M, Johnson SD, Tolley KA, Kiellberg F, van Noort S, Proffit M (2011) Floral volatiles, pollinator sharing and diversification in the fig-wasp mutualism: Insights from Ficus natalensis, and its two wasp pollinators (South Africa). Proceedings of the Royal Society of London B: Biological Sciences, 279, 1731-1739.

Cruaud A, Jabbour-Zahab R, Genson G, Cruaud C, Couloux A, Kjellberg F, van Noort S, Rasplus JY (2010) Laying the foundations for a new classification of Agaonidae (Hymenoptera: Chalcidoidea), a multilocus phylogenetic approach. 
Cladistics, 26, 359-387.

Cruaud A, Rønsted N, Chantarasuwan B, Chou LS, Clement WL, Couloux A, Cousins B, Genson G, Harrison RD, Hanson PE, Hossaert-Mckey M, Jabbour-Zahab R, Jousselin E, Kerdelhué C, Kjellberg F, Lopez-Vaamonde C, Peebles J, Peng YQ, Pereira RAS, Schramm T, Ubaidillah R, van Noort S, Weiblen GD, Yang DR, Yodpinyanee A, Libeskind-Hadas R, Cook JM, Rasplus JY, Savolainen V (2012) An extreme case of plant-insect co-diversification: Figs and fig-pollinating wasps. Systematic Biology, 61, 1029-1047.

Darwell CT, Al-Beidh S, Cook JM (2014) Molecular species delimitation of a symbiotic fig-pollinating wasp species complex reveals extreme deviation from reciprocal partner specificity. BMC Evolutionary Biology, 14, 189.

Galil J, Eisikowitch D (1968) On the pollination ecology of Ficus sycomorus in East Africa. Ecology, 49, 259-269.

Galil J, Eisikowitch D (1969) Further studies on the pollination in Ficus sycomorus L. (Hymenoptera, Clacidoidea, Agaonidae). Tijdschrift Voor Entomologie, 112, 1-13.

Grandi G (1916) Gli Agaonini (Hymenoptera Chalcididae) raccolti nell'Africa occidentale dal Prof. F. Silvestri. Bollettino del Laboratorio di Zoologia Portici, 10, 121-286.

Grandi G (1922) Identification of some fig insects (Hymenoptera) from the British Museum (Natural History). Bulletin of Entomological Research, 13, 295-299.

Grandi G (1927) Hyménopteres sycophiles récoltés aux iles Philippines par CF Baker, I. Agaonini. Philippine Journal of Science, 33, 309-329.

Haine ER, Martin J, Cook JM (2006) Deep mtDNA divergences indicate cryptic species in a fig-pollinating wasp. BMC Evolutionary Biology, 6, 83.

Harrison RD (2005) Figs and the diversity of tropical rainforests. BioScience, 55, 1053-1064.

Hebert PDN, Ratnasingham S, de Waard JR (2003) Barcoding animal life: Cytochrome $c$ oxidase subunit 1 divergences among closely related species. Proceedings of the Royal Society of London B: Biological Sciences, 270, S96-S99.

Hebert PDN, Stoeckle MY, Zemlak TS, Francis CM (2004) Identification of birds through DNA barcodes. PLoS Biology, 2, 1657-1663.

Herre EA, Machado CA, Bermingham E, Nason JD, Windsor DM, McCafferty SS, van Houten W, Bachmann K (1996) Molecular phylogenies of figs and their pollinator wasps. Journal of Biogeography, 23, 521-530.

Janzen DH (1979) How to be a fig. Annual Review of Ecology and Systematics, 10, 13-51.

Johri BM, Konar RN (1955) A contribution to the morphology and embryology of Ficus religiosa Linn. Current Science, 24, 382-385.

Joseph KL (1953) Contributions to our knowledge of fig insects (Chalcidoidea: parasitic Hymenoptera) from India. IV. Descriptions of three new and records of four known species of Agaonini. Agra University Journal of Research, 2, 267-284.

Kerdelhué C, Clainche IL, Rasplus JY (1999) Molecular phylogeny of the Ceratosolen species pollinating Ficus of the subgenus Sycomorus sensu stricto: Biogeographical history and origins of the species-specificity breakdown cases. Molecular Phylogenetics and Evolution, 11, 401-414.

Kerdelhué C, Hochberg ME, Rasplus JY (1997) Active pollination of Ficus sur by two sympatric fig wasp species in West Africa. Biotropica, 29, 69-75.

Lin RC, Yeung CKL, Fong JJ, Tzeng HY, Li SH (2011) The lack of pollinator specificity in a dioecious fig tree: Sympatric fig-pollinating wasps of Ficus septica in Southern Taiwan. Biotropica, 43, 200-207.

Liu GX, Yang DR, Peng YQ, Compton SG (2015) Complementary fruiting phenologies facilitate sharing of one pollinator fig wasp by two fig trees. Journal of Plant Ecology, 8, 197-206.

Liu M (2014) Divergence and Comparative Phylogeography of Ficus pumila and Its Pollinating Wasps (Wiebesia spp.). PhD dissertation, East China Normal University, Shanghai. (in Chinese with English abstract) [刘敏 (2014) 薜荔 (Ficus pumila)及其传粉小蜂(Wiebesia spp.)的分化与比较 亲缘地理学. 博士学位论文, 华东师范大学, 上海.]

Liu M, Zhao R, Chen Y, Zhang J, Compton SG, Chen XY (2014) Competitive exclusion among fig wasps achieved via entrainment of host plant flowering phenology. PLoS ONE, 9, e97783.

Machado CA, Jousselin E, Kjellberg F, Compton SG, Herre EA (2001) Phylogenetic relationships, historical biogeography and character evolution of fig-pollinating wasps. Proceedings of the Royal Society of London B: Biological Sciences, 268, 685-694.

Martinson EO, Jandér KC, Peng YQ, Chen HH, Machado CA, Arnold AE, Herre EA (2014) Relative investment in egg load and poison sac in fig wasps: Implications for physiological mechanisms underlying seed and wasp production in figs. Acta Oecologica, 57, 58-66.

Mayr G (1885) Feigenwespen. Verhandlungen der Zoologisch-Botanischen Gesellschaft in Wien, 35, 147-250.

Michaloud G, Carriere S, Kobbi M (1996) Exceptions to the one : one relationship between African fig trees and their fig wasp pollinators: Possible evolutionary scenarios. Journal of Biogeography, 23, 513-520.

Michaloud G, Michaloud-Pelletier S, Wiebes JT, Berg CC (1985) The co-occurrence of two pollinating species of fig wasp and one species of fig. Proceedings of the Koninklijke Nederlandse Akademie van Wetenscahppen, Series C, 88, 93-119.

Moe AM, Rossi DR, Weiblen GD (2011) Pollinator sharing in dioecious figs (Ficus: Moraceae). Biological Journal of the Linnean Society, 103, 546-558.

Moe AM, Weiblen GD (2010) Molecular divergence in allopatric 
Ceratosolen (Agaonidae) pollinators of geographically widespread Ficus (Moraceae) species. Annals of the Entomological Society of America, 103, 1025-1037.

Molbo D, Krieger MJB, Herre EA, Keller L (2002) Species-diagnostic microsatellite loci for the fig wasp genus Pegoscapus. Molecular Ecology Resources, 2, 440-442.

Molbo D, Machado CA, Sevenster JG, Keller L, Herre EA (2003) Cryptic species of fig-pollinating wasps: Implications for the evolution of the fig-wasp mutualism, sex allocation, and precision of adaptation. Proceedings of the National Academy of Sciences, USA, 100, 5867-5872.

Müller F (1886) Feigenwespen. Kosmos (Stuttgart), 18, 55-62.

Parrish TL, Koelewijn HP, van Dijk PJ, Kruijt M (2003) Genetic evidence for natural hybridization between species of dioecious Ficus on island populations. Biotropica, 35, 333-343.

Peng YQ, Compton SG, Yang DR (2010) The reproductive success of Ficus altissima and its pollinator in a strongly seasonal environment: Xishuangbanna, Southwestern China. Plant Ecology, 209, 227-236.

Peng YQ, Duan ZB, Yang DR, Rasplus JY (2008) Co-occurrence of two Eupristina species on Ficus altissima in Xishuangbanna, SW China. Symbiosis, 45, 9-14.

Pothasin P, Compton SG, Wangpakapattanawong P (2016) Seasonality of leaf and fig production in Ficus squamosa, a fig tree with seeds dispersed by water. PLoS ONE, 11, e0152380.

Ramírez BW (1970a) Taxonomic and biological studies of neotropical fig wasps (Hymenoptera: Agaonidae). The University of Kansas Science Bulletin, 49, 1-44.

Ramírez BW (1970b) Host specificity of fig wasps (Agaonidae). Evolution, 24, 680-691.

Rasplus JY (1996) The one-to-one species-specificity of the Ficus-Agaoninae mutualism: How casual? In: The Biodiversity of African Plants (eds van der Maesen LJG, van der Burgt XM, van Medenbach de Rooy JM), pp. 639-649. Kluwer Academic Publishers, Wageningen.

Rønsted N, Weiblen GD, Clement W, Zerega N, Savolainen V (2008) Reconstructing the phylogeny of figs (Ficus, Moraceae) to reveal the history of the fig pollination mutualisms. Symbiosis, 45, 45-56.

Shanahan M, So S, Compton SG, Corlett R (2001) Fig-eating by vertebrate frugivores: A global review. Biological Reviews, 76, 529-572.

Su Z, Iino H, Nakamura K, Serrato A, Oyama K (2008) Breakdown of the one-to-one rule in Mexican fig-wasp associations inferred by molecular phylogenetic analysis. Symbiosis, 45, 73-81.

Sun XJ, Xiao JH, Cook JM, Feng G, Huang DW (2011) Comparisons of host mitochondrial, nuclear and endosymbiont bacterial genes reveal cryptic fig wasp species and the effects of Wolbachia on host mtDNA evolution and diversity. BMC Evolutionary Biology, 11, 86.
Sutton TL, DeGabriel JL, Riegler M, Cook JM (2017) Local coexistence and genetic isolation of three pollinator species on the same fig tree species. Heredity, 118, 486-490.

Tsai L, Hayakawa H, Fukuda T, Yokoyama J (2015) A breakdown of obligate mutualism on a small island: An interspecific hybridization between closely related fig species (Ficus pumila and Ficus thunbergii) in Western Japan. American Journal of Plant Sciences, 6, 126-131.

Wachi N, Kusumi J, Tzeng HY, Su ZH (2016) Genome-wide sequence data suggest the possibility of pollinator sharing by host shift in dioecious figs (Moraceae, Ficus). Molecular Ecology, 25, 5732-5746.

Warren M, Robertson MP, Greeff JM (2010) A comparative approach to understanding factors limiting abundance patterns and distributions in a fig tree-fig wasp mutualism. Ecography, 33, 148-158.

Weiblen GD (2001) Phylogenetic relationships of fig wasps pollinating functionally dioecious Ficus based on mitochondria DNA sequences and morphology. Systematic Biology, 50, 243-267.

Weiblen GD, Bush GL (2002) Speciation in fig pollinators and parasites. Molecular Ecology, 11, 1573-1578.

Wiebes JT (1963) Taxonomy and host preferences of Indo-Australian fig wasps of the genus Ceratosolen (Agaonidae). Tijdeschrift Voor Entomologie, 106, 1-112.

Wiebes JT (1964) Fig wasps from Israeli Ficus sycomorus and related East African species (Hymenoptera, Chalcidoidea: Agaonidae). Entomologische Berichten Amsterdam, 24, 187-191.

Wiebes JT (1994) The Indo-Australian Agaoninae (pollinators of figs). Verhandelingen der Koninklijke Nederlandsche (Nederlandse) Akademie van Wetenschappen, 92, 1-208.

Wiebes JT, Compton SG (1990) Agaonidae (Hymenoptera Chalcidoidea) and Ficus (Moraceae): Fig wasps and their figs. VI. (Africa concluded). Proceedings of the Koninklijke Nederlandse Akademie van Wetenschappen (C), 93, 203-222.

Xu ZF (1994) Ficus-A keystone plant species in the tropical rain forests ecosystem of south Yunnan. Chinese Biodiversity, 2, 21-23. (in Chinese) [许再富 (1994) 榕树一一滇南 热带雨林生态系统中的一类关键植物. 生物多样性, 2 , 21-23.]

Yang LY, Machado CA, Dang XD, Peng YQ, Yang DR, Zhang DY, Liao WJ (2015) The incidence and pattern of copollinator diversification in dioecious and monoecious fig. Evolution, 69, 294-304.

Zhang DY, Hanski I (1998) Sexual reproduction and stable coexistence of identical competitors. Journal of Theoretical Biology, 193, 465-473.

Zhang DY, Lin K, Hanski I (2004) Coexistence of cryptic species. Ecology Letters, 7, 165-169.

(责任编委: 陈小勇 责任编辑: 时意专) 